\title{
Analysis of motor performance in individuals with cerebral palsy using a non-immersive virtual reality task - a pilot study
}

This article was published in the following Dove Medical Press journal: Neuropsychiatric Disease and Treatment

\author{
Fabiana Paula Almeida \\ Martins ${ }^{1,2}$ \\ Thaís Massetti ${ }^{3}$ \\ Tania Brusque Crocetta' \\ Priscila Bianchi Lopes ${ }^{4}$ \\ Amanda Abreu da Silva ${ }^{2}$ \\ Eliketylen Fernandes \\ Figueiredo ${ }^{2}$ \\ Luiz Carlos de Abreu' \\ Talita Dias da Silva ${ }^{5}$ \\ Carlos Bandeira de Mello \\ Monteiro ${ }^{3}$ \\ 'Study Design and Scientific \\ Writing Laboratory, ABC Medical \\ School (FMABC), Santo André, \\ São Paulo, Brazil; ${ }^{2}$ Research \\ Laboratory, Northern Union of \\ Education (UniNorte), Barão do Rio \\ Branco Faculty, Rio Branco, Acre, \\ Brazil; ${ }^{3}$ Postgraduate Program in \\ Rehabilitation Sciences, Faculty of \\ Medicine, University of São Paulo, \\ São Paulo, São Paulo, Brazil; ${ }^{4}$ Federal \\ University of São Paulo, São Paulo, \\ São Paulo, Brazil; ${ }^{5}$ Department of \\ Medicine, School of Arts, Sciences and \\ Humanities, University of São Paulo, \\ São Paulo, São Paulo, Brazil
}

Correspondence: Thaís Massetti Postgraduate Program in Rehabilitation Sciences, Faculty of Medicine, University of São Paulo, Av. Dr. Arnaldo, 455 Pinheiros, São Paulo, São Paulo, 01246-903, Brazil

Email thaismassetti@gmail.com
Purpose: To evaluate the performance improvement of individuals with hemiparesis cerebral palsy (CP) using a virtual task.

Participants and methods: Twenty individuals were selected and distributed into two groups. The experimental group (CP group) comprised ten individuals with a medical diagnosis of CP, and ten individuals with typical development (sex- and age-matched) composed the control group (TD group). Both groups followed the same intervention protocol, which included a virtual coincident timing task: the participants performed upper limb movements in front of a computer's webcam and interacted with the task with the aim of virtually intercepting spheres that fell in four rows following the rhythm of a pre-selected song during an 8-minute period. To verify the influence on a real task, pre- and posttests were performed in a similar task, but with physical contact (using the spacebar on the keyboard of a computer). To analyze the data, we evaluated the variable, constant, and absolute errors during the task and in the pre- and posttests. Results: The results showed that there was an improvement in performance between the pre- and posttests; that is, after practicing the task in an environment without physical contact, there was a performance improvement in posttests in the real task, but only for the CP group. Moreover, there were significant differences in precision and accuracy between the two groups, with worse performance in the $\mathrm{CP}$ group.

Conclusion: Individuals with CP presented better performance in the real task after practice in a virtual reality task, albeit with worse performance compared with individuals with TD This is an interesting result that supports the possible use of virtual tasks for the rehabilitation of individuals with $\mathrm{CP}$.

Keywords: cerebral palsy, motor performance, virtual reality, motor disability, spastic cerebral palsy

\section{Introduction}

Cerebral palsy (CP) is defined as a group of permanent disorders of movement and posture, resulting from a non-progressive lesion in the brain in the phase of structural growth and functional development. ${ }^{1,2}$ Motor disorders are commonly associated with changes in sensation, learning, body perception, communication, and behavior, in addition to secondary complications such as epilepsy and musculoskeletal disorders that impair the individual's functional performance. ${ }^{1}$

The difficulties in motor function are variable and result in the individual's inability to maintain their posture and perform normal movements, ${ }^{3-6}$ causing limitations in most daily life activities and restricting their social participation. ${ }^{7,8}$ Thus, individuals with CP need to participate in a multidisciplinary rehabilitation program that should 
consider several targets, including environmental and personal factors. ${ }^{9}$ Therefore, the rehabilitation can be considered a long and arduous process for many individuals with disabilities, and as such, clinicians need to face the challenging task of searching for a variety of appealing, meaningful, and motivating intervention tools that may be adapted and graded to facilitate this process. ${ }^{10}$

Under this context, with the growing accessibility of computer-assisted technology, rehabilitation programs increasingly use virtual reality (VR) environments to enhance task practice. ${ }^{11-14}$ Additionally, VR has recently been explored as an intervention specifically to improve motor function in children with $\mathrm{CP} .{ }^{9}$

VR is defined as "the use of interactive simulations created with computer hardware and software to present users with opportunities to engage in environments that appear to be and feel similar to real-world objects and events". ${ }^{10}$ According to the literature, ${ }^{15-17}$ some of the advantages of VR include the ability to practice at home (ie, online), independently or in interaction with others (eg, e-games), and with or without the supervision of a professional. However, it should be noted that VR as an intervention for individuals with CP is relatively new, and although research is rapidly evolving, its benefits and limitations have not been extensively researched. ${ }^{13,18}$

In systematic literature reviews, Chen et $\mathrm{al}^{9,19}$ examined the effect of VR function in children with $\mathrm{CP}$ and found that VR is a viable intervention to improve arm function, ambulation, and postural control. However, the authors also stressed that the reasons why VR works remain unknown and highlighted the need for a more vigorous research design to make a conclusive recommendation.

Despite the positive research using VR in the treatment of individuals with $\mathrm{CP}$, de Mello Monteiro et $\mathrm{al}^{20}$ suggested that this theme should be viewed with some reservations. These authors have examined whether performance improvements in the virtual environment generalize to the real environment in individuals with $\mathrm{CP}$ while performing two coincident timing tasks, one with physical contact and the other without physical contact. The authors found that for individuals with $\mathrm{CP}$, as well as their typically developing peers, coincident timing improved with practice on both tasks. However, the authors also suggested that these improvements were specific to the practice environment, stressed that there was no transfer of learning between environments, and indicated the importance of further research to deeply investigate this issue.

In this sense, it is important to emphasize the distinction between practicing a task in an environment with physical contact (more real characteristics) or without physical contact (more virtual characteristics). In environments without physical contact, participants perform a given task, but the goal is often relatively abstract and directed toward intangible objects, which can directly influence performance. When performing a task in an environment without physical contact, space-time organization differs from that in a real environment, especially among individuals with movement disorders. ${ }^{20,21}$

Given this context, the present work intends to analyze the effect of short-term practice using a non-immersive VR task on the performance of individuals with CP. For this, individuals with CP and with typical development (TD) performed a pretest in a physical contact task and then carried out a similar practice task in a more virtual environment (without physical contact). ${ }^{20,21}$ After practice, the groups returned to the device with physical contact to see if their performance had improved.

Based on CP difficulties such as spasticity, reduced range of motion, and changes in posture and movement, we hypothesized that the CP group would perform poorly compared with the TD group throughout the research protocol; however, because of the difficulty in practicing without physical contact, both groups (CP and TD) would show performance improvement at the moment they perform the task in the posttest (with physical contact).

If the hypothesis is verified, it will offer support for the use of tasks without physical contact (with more virtual features) as a rehabilitation program for individuals with $\mathrm{CP}$, offering a conceptual theoretical basis for future work and clinical interventions.

\section{Participants and methods Participants}

Twenty individuals (children and adolescents) aged 6-19 years were selected (the WHO classifies subjects aged up to 19 years and 364 days as adolescents). ${ }^{22}$ The experimental group consisted of ten individuals with a diagnosis of CP, with spastic hemiparetic functional impairment. The control group consisted of ten individuals with TD. The groups were matched by age and sex, and both were subjected to the same protocol.

The criteria for inclusion in the CP group were individuals with a clinical diagnosis of CP and motor characteristics of mild spastic hemiparesis, classification of gross motor function in stage I or II according to the Gross Motor Function Classification System (GMFCS), ${ }^{23,24}$ and Manual Ability Classification System (MACS) classification level I or II. ${ }^{25}$ 
The following subjects were excluded from the study: those who did not understand the proposed task after three attempts, those with comorbidities that impeded the performance of the activity (skeletal muscle deformity), and individuals who underwent a chemical block or surgery of the upper limbs $<6$ months prior to the study.

This study was approved by the Uninorte Ethics Committee of the Barão do Rio Branco Faculty under protocol 1.996.775 and was conducted in accordance with the Declaration of Helsinki. Participants or their legal representatives (for minors) signed the informed consent form. ${ }^{26}$

\section{Instruments}

Two different software programs were used, one that provided the task of coincident timing in an environment with physical contact (more real characteristics) for the pre- and posttests, and another, "MoveHero", that provided the intervention through a virtual task. Both programs were developed by the Research and Technological Applications in Rehabilitation (Grupo de Pesquisa e Aplicação Tecnológica em Reabilitação) group of the School of Arts, Sciences, and Humanities of the University of Sao Paulo. ${ }^{27}$

\section{Coincident timing software - real task}

A coincident timing task is used to understand the human visuo-motor system, including how motor control processes information involved in intercepting a moving object. ${ }^{27}$ In this study, the coincident timing software used three-dimensional images (Figure 1) and enabled the task to be performed by pressing the space bar on a keyboard. The coincident timing task was based on the Bassin and Reeve ${ }^{28}$ Anticipation Timer and has been used worldwide by many authors ${ }^{20,29,30}$ to assess and verify motor learning based on the performance obtained by decreasing errors or the variability of errors. According to de Mello Monteiro et al, ${ }^{21}$ a decrease in errors in accomplishing the target proposed in the coincident timing task can be considered a performance improvement.

For the coincident timing software, ten spheres that turned on a red light were displayed on the computer screen in sequence until the last sphere was reached, which was considered the target (Figure 1). The participants received immediate feedback on the correctness or error of the task performance, either through different sounds (auditory interaction - differentiated sounds for correctness or error) or through images that changed color (visual interaction - green for correctness and red for an error). The individuals were instructed to place their hand next to the computer keyboard's space bar and were told that when the first/top sphere was activated, they could move their hand when they felt it was appropriate to touch the space bar exactly at the moment the last sphere (target sphere) was activated (lit). This task was applied in the pre- and posttest protocols. It is important to

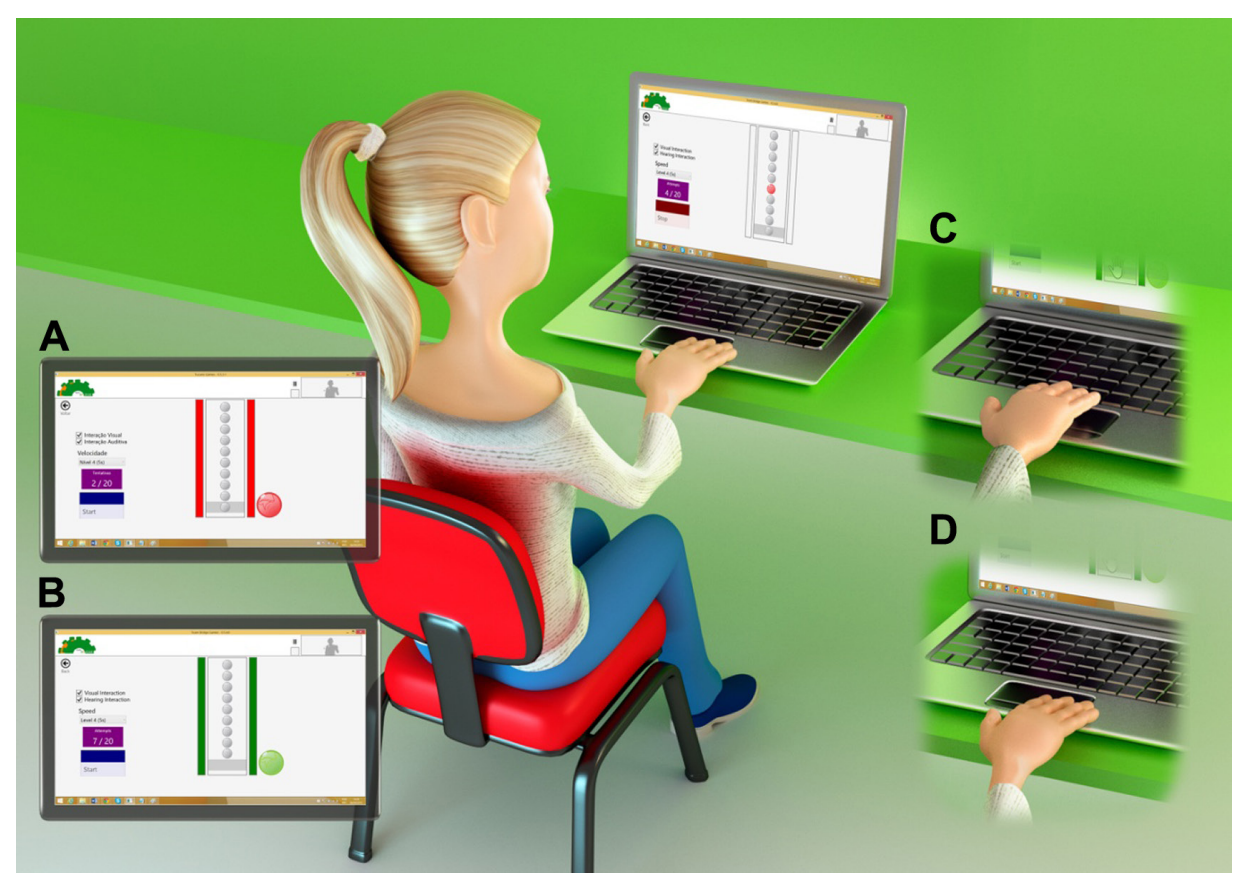

Figure I Illustrative drawing of the coincident timing task.

Notes: (A) Demonstration of error performed by the participant on each attempt (red light - unsuccessful). (B) Demonstration of hit performed by the participant on each attempt (green light - successful). (C) An example of a participant performing the task using the space button of the keyboard. (D) Initial position of the hand. 
emphasize that individuals with $\mathrm{CP}$ were instructed to use the paretic upper limb (limb with greater difficulty) and the control group used the same upper limb as their corresponding peer in the $\mathrm{CP}$ group.

\section{MoveHero software - virtual task}

MoveHero is a game that presents falling spheres on the computer screen, in four imaginary columns, with a musical rhythm selected by the researcher. This is also considered a coincident timing task; the action is to react (using the upper limbs) and not let the balls pass from the fixed targets. The spheres should only be intercepted when they reach the targets allocated in parallel (at two height levels), two on the left (left position targets A and B) and two on the right of the participant (right position targets $C$ and $D$ ), as shown in Figure 2. The virtual contact was performed by the avatar of the individual, that is, a representation of the individual appeared on the computer screen. The individual moved their arms in front of the webcam to coincide with the moment the ball touched the target. The individuals were positioned at a distance of $1.5 \mathrm{~m}$ from the computer monitor and they waited for the balls to drop (which fell randomly on each target). The avatar's hand should reach the target sphere along with the arrival of the ball. Thus, the game requires that the participant has a strategy of anticipation of the movement.

The game offers feedback on correctness and error by means of changing the spheres' color (green for correct and a red line for error) as well as a number $(+1)$ that appears next to the sphere when it is correctly reached inside the target. In addition, in the upper left corner of the screen, the total score of 10 points for each correct task is visible, generating a maximum total of 690 points per song used. The practice time was 8 minutes (four songs of 2 minutes each). In this cross-sectional protocol, no upper limb constriction was used during VR practice, and to allow functionality, coherence, and involvement in the proposed task, the individuals used both upper limbs; that is, the spheres on the right side were to be hit by the right upper limb and those on the left side by the left upper limb.

\section{Procedures and design}

Individuals performed the task individually in a quiet room, with only the experimenter who provided the instructions. The computer was positioned on a desk. The individuals remained seated with the seat height adjusted according to individual needs. The functioning of the game and interfaces (touchscreen for the pre- and posttest and webcam for the practice of the task in a virtual environment) was clarified verbally and by a demonstration.

To match our objective, we used a protocol that allowed us to measure the improvement in coincident timing (accuracy and precision of movement) in a real environment to compare the performance pre- and posttest after practice of a similar task in a virtual environment (ie, all individuals

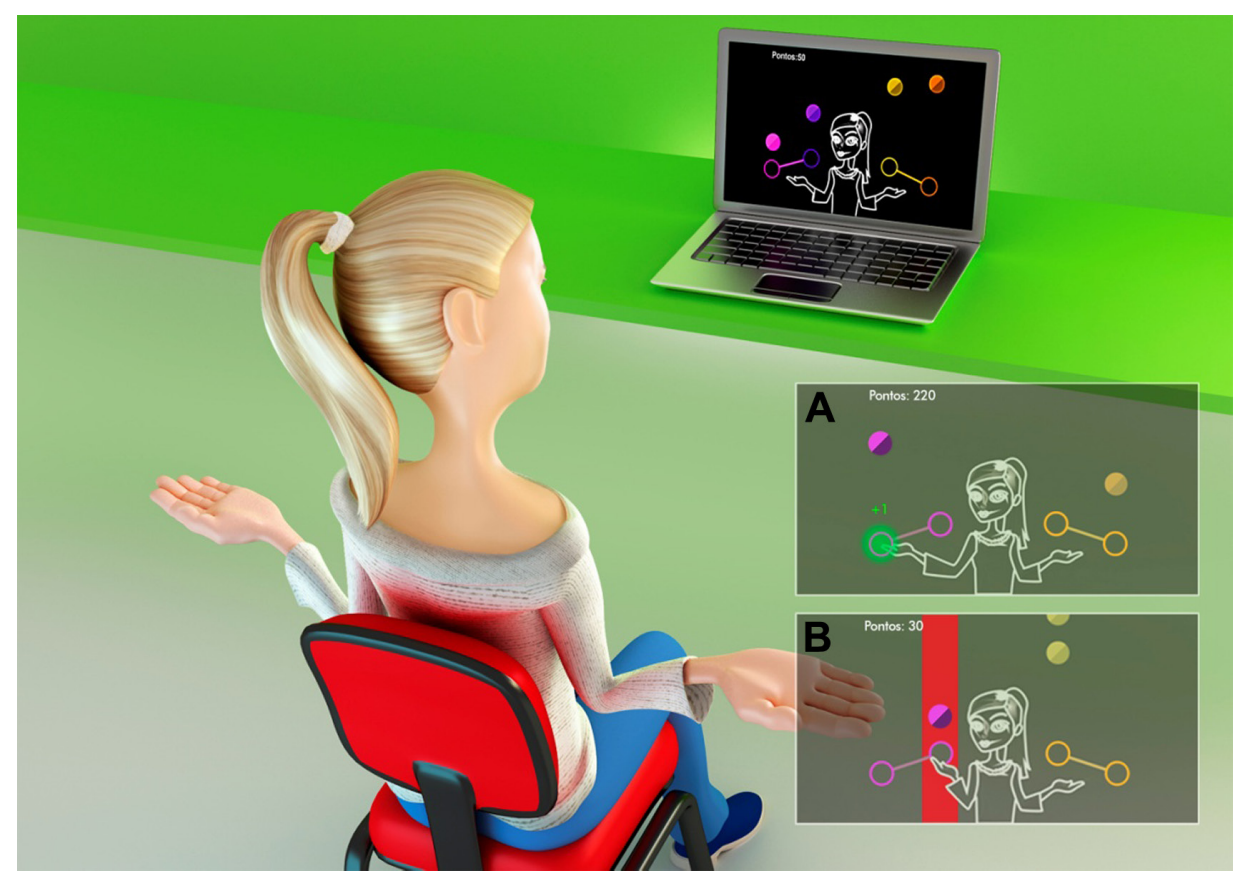

Figure 2 Illustrative drawing of the MoveHero software.

Notes: (A) Demonstration of hit performed by the participant (green light). (B) Error performed by the participant (red light). 


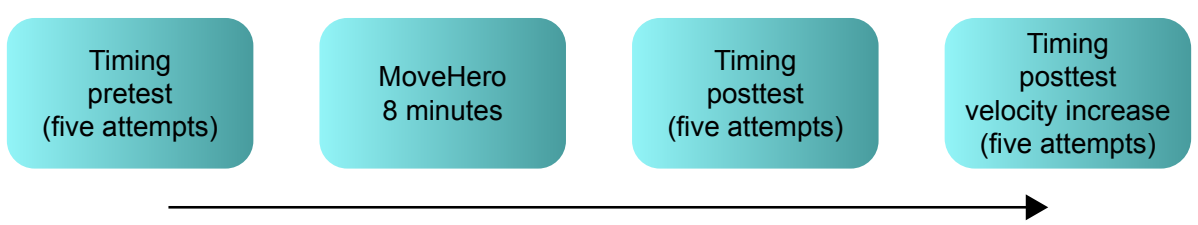

Figure 3 Design of the study.

executed the 1-day protocol with the pretest on a real task followed by virtual task performance and a posttest on the same real task executed previously).

In this protocol, the individuals initially performed a pretest with five attempts at the coincident timing task $(0.5$ seconds velocity between the turning on of each sphere) using the touchscreen (environment with tactile feedback - real) as the interaction device. After the pretest, they all performed the virtual task practice using MoveHero (without tactile feedback) for 8 minutes (four songs of 2 minutes each). Immediately after practice using MoveHero, the individuals performed five more trials in the posttest on the same initial task (with physical contact). Then, we applied a modification of the task to assess performance adaptation (ie, if good performance could be maintained after a change in task). For the modified task, after the first posttest, they performed five more attempts in a second posttest phase with a velocity increase (increase in the velocity of the task of 0.25 seconds between the turning on of each sphere). The design of the study is shown in Figure 3.

\section{Data analysis}

The coincident timing was provided as the time in milliseconds considering the error from the target; that is, the more distant the touch was from the target, the greater the error in milliseconds. The timing error was defined as the difference between the time that the target sphere was turned on and the time that the key was triggered (real coincident timing). In the case of MoveHero (virtual task), the timing error was defined as the difference between the time the ball hit the target sphere and the time the individual managed to hit the target with the avatar's hand. As used by de Mello Monteiro et al, ${ }^{20,21}$ Quadrado et al, ${ }^{31}$ and Bezerra et al, ${ }^{32}$ the error time in milliseconds can be used to analyze the constant error (CE), which evaluates the directional tendency of the movement; the absolute error (AE), which demonstrates the accuracy of the movement; and the variable error (VE), which identifies the accuracy of the movement (for details about these errors, see de Mello Monteiro et $\mathrm{a}^{21}$ ). $\mathrm{CE}, \mathrm{AE}$, and VE were used as dependent variables for both the coincident timing task and MoveHero.

For the coincident timing task, the results were obtained using blocks (average of five trials in each) for all phases of the study (pretest, posttest, and posttest with a velocity increase). For the MoveHero task, the mean number of attempts for each position of the balls (the mean of positions $\mathrm{A}$ and $\mathrm{B}$ was considered the left position, and the mean of positions $\mathrm{C}$ and $\mathrm{D}$ was considered the right position) was obtained. The dependent variables (CE, AE, and VE) were subjected to one-way ANOVA with the following factors: two between-group factors (CP and TD) and two withinblock factors, with repeated measures in the last factor (blocks). In the block factor for the coincident timing task, separate comparisons were made for the study phases: pre- vs posttest and pre- vs posttest with a velocity increase. In the MoveHero task, to assess the effect of training (improvement of performance with practice) in both groups, separate comparisons were made for the different songs: song 1 (S1) vs song 2 (S2); S2 vs song 3 (S3); and S3 vs song 4 (S4), as well as for the different positions (left and right). Post hoc comparisons were performed with the least significant difference test. Partial $\eta^{2}$ was used to calculate the effect size, where $\eta_{\mathrm{p}}{ }^{2}=0.01$ was considered a small effect, $\eta_{\mathrm{p}}{ }^{2}=0.06$ a moderate effect, and $\eta_{p}{ }^{2}=0.14$ a large effect. ${ }^{33}$ Values of $P<0.05$ were considered significant. The statistical package used was SPSS (IBM Corporation, Armonk, NY, USA), version 20.0.

\section{Results}

The coincident timing task was applied in three different phases to evaluate the effect of practice on the VR task: 1) in the pretest phase, five attempts at the velocity of 0.5 seconds between the turning on of the spheres; 2) in the posttest phase, five trials at the same velocity as the pretest; and 3) in the posttest phase, five trials with a 0.25 -second velocity increase (VI) between the turning on of the spheres.

\section{Coincident timing - pre- vs posttest}

The results were obtained using blocks (five trials each) for all phases of the study (pretest, posttest, and posttest with VI).

\section{Constant error}

The CE analyzes the anticipation or delay in the reaction in the assigned task; in this case, this was conducted by comparing the pre- and posttest phases of the coincident timing 


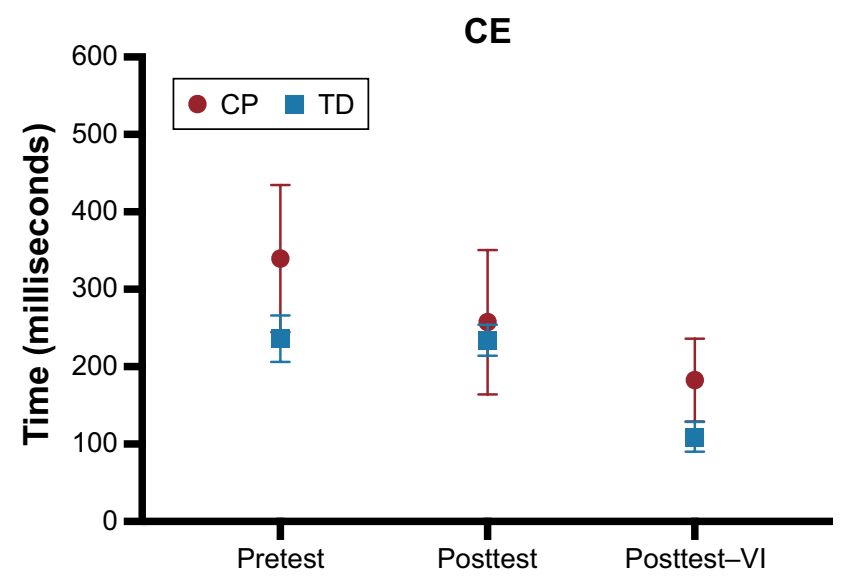

Figure 4 Mean and standard error representation of the CE of the CP and TD groups in all phases of the experiment.

Abbreviations: CE, constant error; CP, cerebral palsy; TD, typical development; $\mathrm{VI}$, velocity increase.

interface. ANOVA did not indicate significant effects or interactions for blocks or groups. These results indicate that both groups showed a tendency to delay the contact, without significant differences from the start to the end of the test. The mean and standard error values are shown in Figure 4.

\section{Absolute error}

For the AE, there were significant effects of blocks $\left(F_{1,15}=5.01, P=0.041, \eta_{p}^{2}=0.25\right)$ and groups $\left(F_{1,15}=7.83\right.$, $\left.P=0.014, \eta_{p}^{2}=0.34\right)$ and a marginally significant interaction between blocks and groups $\left(F_{1,15}=4.28, P=0.056, \eta_{p}^{2}=0.22\right)$, as shown in Figure 5. These results indicate that there was performance improvement between the pre- and posttests; that is, after practicing the MoveHero task, performance improvement occurred. However, the post hoc test indicated that this improvement in performance was significant only for the $\mathrm{CP}$ group. In addition, the $\mathrm{CP}$ group had a worse $\mathrm{AE}$

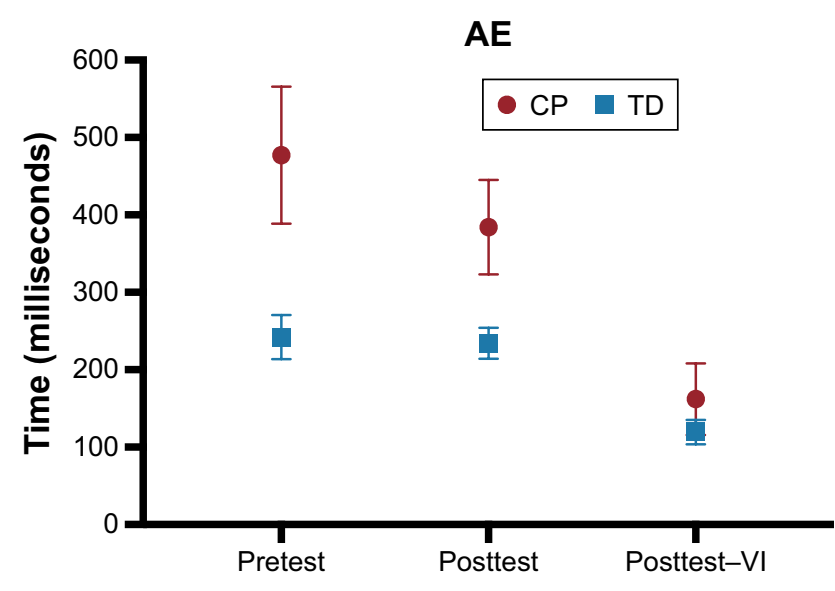

Figure 5 Mean and standard error representation of the AE of the CP and TD groups in all phases of the experiment.

Abbreviations: $\mathrm{AE}$, absolute error; $\mathrm{CP}$, cerebral palsy; TD, typical development; $\mathrm{VI}$, velocity increase. than the TD group. The mean and standard error values are shown in Figure 5.

\section{Variable error}

Similar to the AE, there were significant differences for blocks $\left(F_{1,15}=6.22, P=0.025, \eta_{p}^{2}=0.29\right)$ and groups $\left(F_{1,15}=6.23, P=0.025, \eta_{p}^{2}=0.29\right)$ regarding VE. These results show that there was an improvement in the VE between the pre- and posttests, and the CP group presented a worse VE when compared with the TD group (Figure 6).

\section{Coincident timing - pre- vs posttest with a velocity increase}

\section{Constant error}

ANOVA showed a significant effect for blocks $\left(F_{1,16}=9.09\right.$, $P=0.008, \eta_{p}^{2}=0.36$ ), without an effect for groups, as well as no significant interaction between blocks and groups. These results indicate that both groups showed a tendency to delay the contact and that both groups improved the CE from the pretest to posttest with VI. The mean and standard error values are shown in Figure 4.

\section{Absolute error}

For the AE, there were significant differences between blocks $\left(F_{1,16}=20.2, P<0.001, \eta_{p}^{2}=0.56\right)$, marginally significant differences between groups $\left(F_{1,16}=4.29, P=0.055, \eta_{p}^{2}=0.21\right)$, and a significant interaction between blocks and groups $\left(F_{1,16}=4.57, P=0.048, \eta_{p}^{2}=0.22\right)$, as shown in Figure 5. These results indicate that there was a performance improvement between the pre- and posttest with VI; however, the post hoc test indicated that this performance improvement was significant only for the $\mathrm{CP}$ group. In addition, the $\mathrm{CP}$ group presented a worse $\mathrm{AE}$ than the TD group only in the

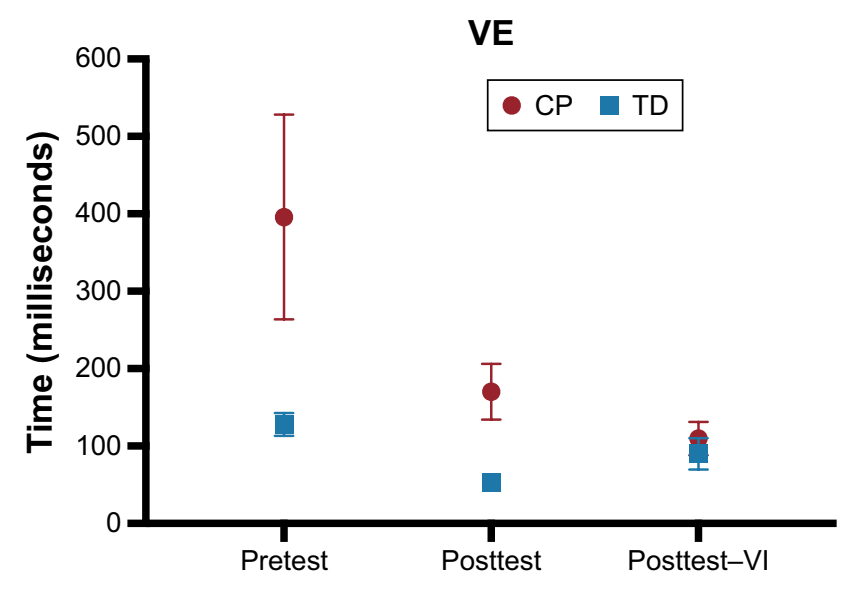

Figure 6 Mean and standard error representation of the VE of the CP and TD groups in all phases of the experiment.

Abbreviations: CP, cerebral palsy; TD, typical development; VE, variable error; $\mathrm{VI}$, velocity increase. 

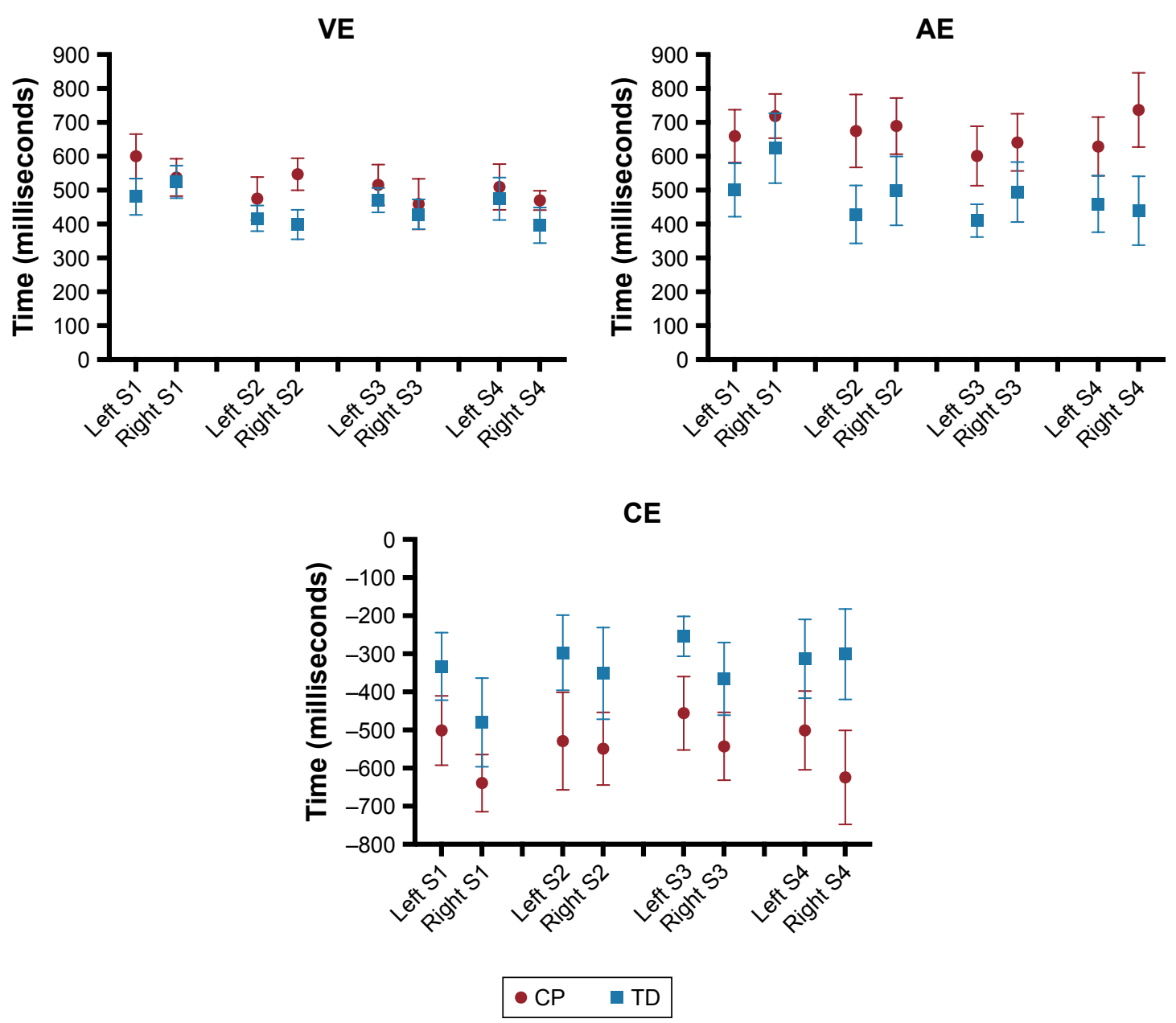

Figure 7 Mean and standard error representation of VE, AE, and CE of both CP and TD groups in all phases of the experiment.

Notes: 'Left' represents positions of the balls $\mathrm{A}$ and $\mathrm{B}$ together and 'Right' represents positions $\mathrm{C}$ and $\mathrm{D}$ together. " $\mathrm{S}$ " represents each song they played. There were 4 songs and they are represented as SI (song I), S2 (song 2), S3 (song 3), and S4 (song 4).

Abbreviations: $\mathrm{AE}$, absolute error; $\mathrm{CE}$, constant error; $\mathrm{CP}$, cerebral palsy; TD, typical development; $\mathrm{VE}$, variable error.

pretest block. The mean and standard error values are shown in Figure 5.

\section{Variable error}

A significant difference was found for blocks $\left(F_{1,16}=5.58\right.$, $\left.P=0.031, \eta_{p}^{2}=0.26\right)$, and a marginal difference was found for groups $\left(F_{1,16}=3.69, P=0.073, \eta_{p}^{2}=0.19\right)$. These results show that there was an improvement in the VE from pretest to posttest in both groups, and the group with CP had a worse VE when compared with the TD group, but only in the pretest block (Figure 6).

\section{MoveHero}

The results obtained during the MoveHero task performed between the pre- and posttest phases are shown below.

\section{Song I vs song 2}

There were no significant effects or interactions between $\mathrm{S} 1$ and $\mathrm{S} 2$ for the $\mathrm{CE}$ or $\mathrm{AE}$; however, there was an effect for the $\operatorname{VE}\left(F_{1,18}=6.13, P=0.023, \eta_{p}^{2}=0.25\right)$, as $\mathrm{VE}$ was lower for
$\mathrm{S} 2(\mathrm{M}=446 \mathrm{~ms})$ than for S1 $(\mathrm{M}=540 \mathrm{~ms})$. No effects were found for groups (Figure 7).

When comparing the right and left positions of the falling spheres, no significant effects were found for positions or groups (Figure 7).

\section{Song 2 vs song 3}

ANOVA did not find any significant effects for songs, groups, or positions of the ball.

\section{Song 3 vs song 4}

Similar to the comparison between S2 and S3, there were no significant effects for songs, groups, or positions of the ball.

\section{Discussion}

The present study proposed to evaluate performance improvement in individuals with $\mathrm{CP}$ and TD during practice in a non-immersive virtual environment (task without physical contact), by comparing pre- and posttest performance in a real task (with physical contact), in order to verify whether 
practicing a virtual task provides benefits in a similar task in a real environment.

The results showed that there was an improvement in performance between the pre- and posttests; that is, after practicing the task in an environment without physical contact, provided by MoveHero, there was a performance improvement in both posttests (posttest 1 after practice and posttest 2 with a velocity increase) in the real task, but only for the CP group. This is an interesting result that leads to the possible use of virtual tasks for the rehabilitation of individuals with $\mathrm{CP}$.

In this sense, Golomb et $\mathrm{al}^{34}$ conducted a pilot study with individuals with hemiplegic $\mathrm{CP}$ and found that the use of video game therapies can cause significant gains in the function of the paretic upper limb. Additionally, Weightman et $\mathrm{al}^{6}$ and Preston and Ehrsson ${ }^{35}$ highlighted that VR is a valuable tool to improve upper limb functions, whether the VR is used at home or in a school context. Their results pointed to significant improvements in arm kinematics. Using functional magnetic resonance imaging, You et $\mathrm{al}^{36}$ verified that there is a development of new motor skills in the paretic limb of children with $\mathrm{CP}$ after practicing a virtual task.

Although it was not the goal and cannot be verified in this pilot study, the processes of functional improvement induced by VR may be linked to neural modifications, which should encourage future work with VR in CP. Shin et $\mathrm{al}^{37}$ verified changes in neural plasticity related to a virtual task as part of rehabilitation therapy in individuals with CP. Furthermore, Collange Grecco et $\mathrm{al}^{38}$ compared the effects of anodal vs sham transcranial direct current stimulation (tDCS) combined with VR training for improving gait in children with CP. Their results showed that after the intervention with active tDCS associated with VR, children with CP had better performance regarding gait velocity, cadence, gross motor function, and independent mobility compared with the children who underwent sham tDCS.

Could you please change this sentence to: It is worth mentioning another interesting point of the results obtained with the use of VR in this work. The CP group seemed to have lower accuracy and movement time compared with the TD group during the coincident timing practice, which was expected and was observed in previous research studies ${ }^{20,39}$ but during the MoveHero practice, this difference was not observed. Considering that in the MoveHero task, both the CP and TD groups performed similarly, we can speculate the following:

1. Level of function: The recruited individuals with $C P$ had a mild level of change in gross motor function (GMFCS and MACS levels I and II). Although they presented inferior performance compared with the control group in the real coincident timing (with physical contact), they were able to adapt to the virtual task. Chen et $\mathrm{al}^{40}$ found that individuals with CP (hemiplegic) who presented less damage in functionality and a better understanding of the task favored performance improvement, presenting better results after an intervention through VR. In the study by Do et al, ${ }^{41} \mathrm{VR}$ programs were found to be capable of changing motor skills (motor performance) in individuals with hemiplegic CP. Likewise, Pourazar et $\mathrm{al}^{42}$ showed significant improvements in a group of children with $\mathrm{CP}$ after an intervention program using VR.

2. Engagement: The practice of a more motivating task probably provided greater interest to individuals with $\mathrm{CP}$, and in this way, they managed to obtain values close to those of the individuals with TD during the MoveHero practice. Although engagement appeared to be the same for both groups, the TD group (due to their normal pattern of movement) already had great performance from the beginning of the practice, probably because it was a very easy task for them. As a result, the engagement only maintained the good performance of the TD group. Other studies used a motor learning protocol and only used the coincident timing as a laboratory task, which increased the difficulty of engagement and made it impossible to practice for a long period of time..$^{20,31}$ However, the task proposed by the MoveHero software provides a greater engagement, probably due to the number of spheres, songs used, and the existence of an avatar. In this sense, it can be speculated that the involvement and engagement of CP individuals is an essential factor in the use of VR in rehabilitation. The greater the child's motivation to perform the task was, the greater the level of engagement during practice and the better the performance during the task. ${ }^{10,43}$ Other studies have reinforced that the motivation of children with CP promotes greater engagement and emphasized that motivation and engagement are essential factors of VR. ${ }^{9,19,44,45}$

3. In this context, Harris and Roxborough ${ }^{43}$ explored the degree of motivation of 16 children with CP during VR play sessions. The Pediatric Volitional Questionnaire was used to measure children's motivation. Different VR environments produced varying levels of volitional behavior, and the features of environments that produced higher levels of volition included challenge, variability, and competition. The authors highlighted that the overall volitional scores of children with $\mathrm{CP}$ indicated that VR play is a motivating activity and thus has potential as a successful intervention tool. 
Another factor that may have contributed to engagement during the virtual task and provided similar performance between CP and TD groups in this pilot protocol was the use by MoveHero of songs during practice. As noted by Grahn, ${ }^{46}$ music is organized in time and has rhythmic beats that occur at predictable and consistent intervals. This quality allows music to be used as a marker of time and rhythm, thus providing suggestions for the predictability of motor movements. ${ }^{46}$ Therefore, the musical rhythm could become an auditory cue to engage cortical control of movement, which in turn might potentially enhance motor movement. ${ }^{47}$ Furthermore, the literature has suggested that music may support training because the sequential and rhythmic structures of music facilitate the planning and training of the performance of the required movements, in addition to promoting a relaxed and positive emotional state during the VR movements. ${ }^{48}$

In addition, with respect to motor function in $\mathrm{CP}$, the literature has identified a link between music and body reactions for rehabilitation purposes. Music is known to increase mobilization of the proposed activity by increasing concentration, such that the beat of the music itself coincides with the increased performance of the patient. ${ }^{20}$ In terms of coordination, the synchronization between the sounds and gestures induced by music has also been successfully used to teach brain-damaged patients to improve their performance of movements. ${ }^{49}$

4. Patterns of movement: Another possibility is that when us device that needed more global movements with the participation of more proximal movement patterns, participants with CP were able to adapt more to the task and, therefore, presented similar performance to that of the group with TD. That is, the coincident timing with the use of the keyboard required fine control of functions, and thus, there was a greater accuracy of movement. de Mello Monteiro et $\mathrm{al}^{20}$ used the same keyboard task with individuals with $\mathrm{CP}$ and found similar results, with the TD group always showing better performance.

Fernani et $\mathrm{al}^{50}$ compared the accuracy and velocity of movement in individuals with $\mathrm{CP}$ and found that there is greater difficulty in movement during tasks that require more precision of movement than velocity. We can speculate that coincident timing with the use of the keyboard needs more precision and fine movement, while MoveHero has the characteristic of velocity with overall movements. Moreover, in the keyboard task, the individuals with $\mathrm{CP}$ had to control their movement to touch the space bar, which requires coordination, acceleration, and deceleration of the movement. By contrast, for the required movement using a webcam, the CP individuals only had to wave their hands in front of the camera in the correct places to achieve the task, which requires more velocity than fine movements and coordination, corroborating the findings of Fernani et al..$^{50}$ Thus, this better functionality in velocity and overall movements may have been responsible for the best performance in the MoveHero task. ${ }^{50}$ In this sense, it should be noted that such findings reinforce what has been reported in the literature, which indicates that the execution of overall movements is easier to perform when compared with the execution of finer movements in individuals with $\mathrm{CP} .{ }^{50-52}$

In this context, our results reinforce the findings of Gaillard et al, ${ }^{52}$ who noted that in individuals with $\mathrm{CP}$, kinematic abnormalities were more pronounced in the distal segments (elbow flexion-extension, pronosupination, and wrist flexion-extension). The authors indicated that this finding may be related to muscle weakness and to increased muscle tone being more prominent distally and having a higher influence on distal movements.

Furthermore, compensatory movement is frequently observed and may be accompanied by poor functional outcomes in individuals with $\mathrm{CP} .{ }^{53}$ However, despite the difficulties related to these patterns of movement, here we reinforced the functional capability, which may have allowed the participants in the present study to achieve the task's goals in the virtual task even using compensatory movement. ${ }^{54,55}$ Thus, the functional movement of the upper extremity to achieve a virtual target may have positively impacted performance and enhanced functional capability.

Despite the interesting results found in this research, we emphasize that this was a pilot project, and thus, the results should be considered carefully in light of certain limitations. First, the greatest limitation is certainly the small number of participants, which diminished the sensitivity of the tests (ie, some marginal differences were observed between the groups during songs and for the positions of the falling spheres - see Figure 7 - but due to the number of participants, these differences were not significant). A second limitation is that the protocol was short term without any familiarization (only demonstration and verbal explanation), which could have influenced the results. To address this limitation, a long-term protocol (training study) should be organized. The third limitation is the lack of a crossover design. In such a study, a group of individuals with CP would begin with VR training, while another group began with real training; after a period of interventions, both groups would change practices, which would provide a better understanding of the influence 
of VR on CP. The fourth limitation was the necessity of other clinical assessments, such as visual and cognitive assessments, to better characterize the sample and understand the results. Fifth, we did not control compensatory movements, and this could have influenced the results. However, as our focus was on task improvement, future studies aiming at qualitative assessment should examine the influence of patterns of movement during the VR task in individuals with CP. Moreover, considering the above limitations, further studies with a greater number of participants organized with a long-term crossover protocol are needed to verify whether independent variables such as gender, hemiplegic side, age, or functional classification influence the results.

\section{Conclusion}

Individuals with CP presented better performance after practicing a VR task, albeit with worse performance than individuals with TD. Therefore, based on the VR characteristics of motivation, engagement, and effectiveness, VR may be effective for promoting motor performance improvement of individuals with $\mathrm{CP}$.

\section{Ethics approval and informed consent}

The research project and the terms of informed consent for participants were analyzed and approved by the Uninorte Ethics Committee of the Barão do Rio Branco Faculty according to protocol no 1.996 .775 .

\section{Acknowledgments}

The authors would like to acknowledge Ian de Araújo Barros, Maithê Blaya Leite, Fyamma Rillari Trajana Beiruth, Ingrid Guimarães Brandão, Jéssica Raiany Silva Duarte, Leyryana Conceição de Oliveira, Kássia Hianara Sena de Oliveira, and Laysa Cristina Moreira dos Santos for assistance with data collection for this research. This work was supported by the Brazilian agency CNPq (National Counsel of Technological and Scientific Development) under process number 442456/2016-6 and by Convênio Sesacre-FMABC (Faculdade de Medicina do ABC) under process number $007 / 2015$.

\section{Author contributions}

All authors contributed toward data analysis, drafting and critically revising the paper, gave final approval of the version to be published, and agreed to be accountable for all aspects of the work.

\section{Disclosure}

The authors report no conflicts of interest in this work.

\section{References}

1. Bax M, Goldstein M, Rosenbaum P, et al. Proposed definition and classification of cerebral palsy, April 2005. Dev Med Child Neurol. 2005;47(8):571-576.

2. Colver A, Fairhurst C, Pharoah POD. Cerebral palsy. Lancet. 2014; 383(9924):1240-1249.

3. Odding E, Roebroeck ME, Stam HJ. The epidemiology of cerebral palsy: incidence, impairments and risk factors. Disabil Rehabil. 2006;28(4):183-191.

4. Vukojević M, Soldo I, Granić D. Risk factors associated with cerebral palsy in newborns. Coll Antropol. 2009;33(Suppl 2):199-201.

5. Hnatyszyn G, Cyrylowski L, Czeszynska MB, et al. The role of magnetic resonance imaging in early prediction of cerebral palsy. Turk J Pediatr. 2010;52(3):278-284.

6. Weightman A, Preston N, Levesley M, et al. Home based computerassisted upper limb exercise for young children with cerebral palsy: a feasibility study investigating impact on motor control and functional outcome. J Rehabil Med. 2011;43(4):359-363.

7. Tsai $\mathrm{P}$, Yang $\mathrm{T}$, Chan R, Huang P, Wong T. Functional investigation in children with spina bifida - measured by the pediatric evaluation of disability inventory (PEDI). Child's Nerv Syst. 2002;18(1-2):48-53.

8. Kułak W, Okurowska-Zawada B, Sienkiewicz D, Paszko-Patej G, Krajewska-Kułak E. Risk factors for cerebral palsy in term birth infants. Adv Med Sci. 2010;55(2):216-221.

9. Chen Y, Fanchiang HD, Howard A. Effectiveness of virtual reality in children with cerebral palsy: a systematic review and meta-analysis of randomized controlled trials. Phys Ther. 2018;98(1):63-77.

10. Weiss PL, Rand D, Katz N, Kizony R. Video capture virtual reality as a flexible and effective rehabilitation tool. J Neuroeng Rehabil. 2004;1(1):12.

11. Barton GJ, Hawken MB, Foster RJ, Holmes G, Butler PB. The effects of virtual reality game training on trunk to pelvis coupling in a child with cerebral palsy. J Neuroeng Rehabil. 2013;10(1):15.

12. Burdea GC, Cioi D, Kale A, Janes WE, Ross SA, Engsberg JR. Robotics and gaming to improve ankle strength, motor control, and function in children with cerebral palsy - a case study series. IEEE Trans Neural Syst Rehabil Eng. 2013;21(2):165-173.

13. Mitchell L, Ziviani J, Oftedal S, Boyd R. The effect of virtual reality interventions on physical activity in children and adolescents with early brain injuries including cerebral palsy. Dev Med Child Neurol. 2012;54(7): 667-671.

14. Riener R, Dislaki E, Keller U, Koenig A, van Hedel H, Nagle A. Virtual reality aided training of combined arm and leg movements of children with CP. Stud Health Technol Inform. 2013;184:349-355.

15. Hurkmans HL, van den Berg-Emons RJ, Stam HJ. Energy expenditure in adults with cerebral palsy playing Wii sports. Arch Phys Med Rehabil. 2010;91(10):1577-1581.

16. Huber M, Rabin B, Docan C, Burdea GC, Abdelbaky M, Golomb MR. Feasibility of modified remotely monitored in-home gaming technology for improving hand function in adolescents with cerebral palsy. IEEE Trans Inf Technol Biomed. 2010;14(2):526-534.

17. Vissers M, van den Berg-Emons R, Sluis T, Bergen M, Stam H, Bussmann $\mathrm{H}$. Barriers to and facilitators of everyday physical activity in persons with a spinal cord injury after discharge from the rehabilitation centre. J Rehabil Med. 2008;40(6):461-467.

18. Snider L, Majnemer A, Darsaklis V. Virtual reality as a therapeutic modality for children with cerebral palsy. Dev Neurorehabil. 2010; 13(2):120-128.

19. Chen YP, Lee SY, Howard AM. Effect of virtual reality on upper extremity function in children with cerebral palsy: a meta-analysis. Pediatr Phys Ther. 2014;26(3):289-300. 
20. de Mello Monteiro CB, Massetti T, da Silva TD, et al. Transfer of motor learning from virtual to natural environments in individuals with cerebral palsy. Res Dev Disabil. 2014;35(10):2430-2437.

21. de Mello Monteiro CB, da Silva TD, de Abreu LC, et al. Short-term motor learning through non-immersive virtual reality task in individuals with Down syndrome. BMC Neurol. 2017;17(1):71.

22. World Health Organization W. The Health of Youth. Geneva: World Health Organization; 1989.

23. Palisano R, Rosenbaum P, Walter S, Russell D, Wood E, Galuppi B. Development and reliability of a system to classify gross motor function in children with cerebral palsy. Dev Med Child Neurol. 1997;39(4): 214-223.

24. Shevell MI, Dagenais L, Hall N; REPACQ Consortium. Comorbidities in cerebral palsy and their relationship to neurologic subtype and GMFCS level. Neurology. 2009;72(24):2090-2096.

25. Eliasson A-C, Krumlinde-Sundholm L, Rösblad B, et al. The Manual Ability Classification System (MACS) for children with cerebral palsy: scale development and evidence of validity and reliability. Dev Med Child Neurol. 2006;48(07):549-554.

26. Masseti T, Crocetta TB, Guarnieri R, et al. A didactic approach to presenting verbal and visual information to children participating in research protocols: the comic book informed assent. Clinics. 2018;73:e207.

27. Crocetta TB, de Araújo LV, Guarnieri R. Virtual reality software package for implementing motor learning and rehabilitation experiments. Virtual Real. 2018;22(3):199-209.

28. Hart MA, Reeve TG. A preliminary comparison of stimulus presentation methods with the Bassin anticipation timing task. Percept Mot Skills. 1997;85(1):344-346.

29. Chiviacowsky S, Drews R. Temporal-comparative feedback affects motor learning. J Mot Learn Dev. 2016;4(2):208-218.

30. Malheiros SR, da Silva TD, Favero FM, et al. Computer task performance by subjects with Duchenne muscular dystrophy. Neuropsychiatr Dis Treat. 2016;12:41-48.

31. Quadrado VH, Silva TDD, Favero FM, Tonks J, Massetti T, Monteiro CBM. Motor learning from virtual reality to natural environments in individuals with Duchenne muscular dystrophy. Disabil Rehabil Assist Technol. 2017:1-9.

32. Bezerra ÍMP, Crocetta TB, Massetti T, et al. Functional performance comparison between real and virtual tasks in older adults: a crosssectional study. Medicine. 2018;97(4):e9612.

33. Silva-Filho E, Okano AH, Morya E, et al. Neuromodulation treats Chikungunya arthralgia: a randomized controlled trial. Sci Rep. 2018; 8(1): 16010 .

34. Golomb MR, Mcdonald BC, Warden SJ, et al. In-home virtual reality videogame telerehabilitation in adolescents with hemiplegic cerebral palsy. Arch Phys Med Rehabil. 2010;91(1):1-8.

35. Preston $\mathrm{C}$, Ehrsson HH. Illusory changes in body size modulate body satisfaction in a way that is related to non-clinical eating disorder psychopathology. PLoS One. 2014;9(1):e85773.

36. You SH, Jang SH, Kim YH, Kwon YH, Barrow I, Hallett M. Cortical reorganization induced by virtual reality therapy in a child with hemiparetic cerebral palsy. Dev Med Child Neurol. 2005;47(9):628-635.

37. Shin JW, Song GB, Hwangbo G. Effects of conventional neurological treatment and a virtual reality training program on eye-hand coordination in children with cerebral palsy. J Phys Ther Sci. 2015;27(7): 2151-2154.

38. Collange Grecco LA, de Almeida Carvalho Duarte N, Mendonça ME, Galli M, Fregni F, Oliveira CS. Effects of anodal transcranial direct current stimulation combined with virtual reality for improving gait in children with spastic diparetic cerebral palsy: a pilot, randomized, controlled, double-blind, clinical trial. Clin Rehabil. 2015;29(12): $1212-1223$.
39. Prado MTA, Fernani DCGL, Silva TD, Smorenburg ARP, Abreu LC, Monteiro CBM. Motor learning paradigm and contextual interference in manual computer tasks in individuals with cerebral palsy. Res Dev Disabil. 2017;64:56-63.

40. Chen YP, Kang LJ, Chuang TY, et al. Use of virtual reality to improve upper-extremity control in children with cerebral palsy: a single-subject design. Phys Ther. 2007;87(11):1441-1457.

41. Do JH, Yoo EY, Jung MY, Park HY. The effects of virtual reality-based bilateral arm training on hemiplegic children's upper limb motor skills. Neuro Rehabilitation. 2016;38(2):115-127.

42. Pourazar M, Mirakhori F, Hemayattalab R, Bagherzadeh F. Use of virtual reality intervention to improve reaction time in children with cerebral palsy: a randomized controlled trial. Dev Neurorehabil. 2018; 21(8):515-520.

43. Harris SR, Roxborough L. Efficacy and effectiveness of physical therapy in enhancing postural control in children with cerebral palsy. Neural Plast. 2005;12(2-3):229-243.

44. Levac D, Rivard L, Missiuna C. Defining the active ingredients of interactive computer play interventions for children with neuromotor impairments: a scoping review. Res Dev Disabil. 2012;33(1):214-223.

45. Tachihara S, Yamaguchi T, Ishiura N, et al. Characterization of engagement changes during VR based rehabilitation: a preliminary study. Paper presented at: ITE Technical Report 40.11; 2016.

46. Grahn JA. Neural mechanisms of rhythm perception: current findings and future perspectives. Top Cogn Sci. 2012;4(4):585-606.

47. Rochester L, Baker K, Hetherington V, et al. Evidence for motor learning in Parkinson's disease: acquisition, automaticity and retention of cued gait performance after training with external rhythmical cues. Brain Res. 2010;1319:103-111.

48. Trobia J, Gaggioli AA, Antonietti AA. Combined use of music and virtual reality to support mental practice in stroke rehabilitation. J Cyber Ther Rehabil. 2011;4(1):57-61.

49. Gervin AP. Music therapy compensatory technique utilizing song lyrics during dressing to promote independence in the patient with a brain injury. Music Ther Perspect. 1991;9(1):87-90.

50. Fernani D, Prado MTA, da Silva TD, et al. Evaluation of speed-accuracy trade-off in a computer task in individuals with cerebral palsy: a crosssectional study. BMC Neurol. 2017;17(1):143.

51. Figueiredo B, Uncles M. Moving across time and space: temporal management and structuration of consumption in conditions of global mobility. Consump Mark Cult. 2015;18(1):39-54.

52. Gaillard F, Cretual A, Cordillet S, et al. Kinematic motion abnormalities and bimanual performance in children with unilateral cerebral palsy. Dev Med Child Neurol. 2018;60(8):839-845.

53. Fitoussi F, Diop A, Maurel N, Laasel ELM, Ilharreborde B, Penneçot GF. Upper limb motion analysis in children with hemiplegic cerebral palsy: proximal kinematic changes after distal botulinum toxin or surgical treatments. J Child Orthop. 2011;5(5):363-370.

54. Howcroft J, Klejman S, Fehlings D, et al. Active video game play in children with cerebral palsy: potential for physical activity promotion and rehabilitation therapies. Arch Phys Med Rehabil. 2012;93(8): 1448-1456.

55. Berry T, Howcroft J, Klejman S, Fehlings D, Wright V, Biddiss E. Variations in movement patterns during active video game play in children with cerebral palsy. J Bioeng Biomed Sci. 2012;S1:001. 


\section{Publish your work in this journal}

Neuropsychiatric Disease and Treatment is an international, peerreviewed journal of clinical therapeutics and pharmacology focusing on concise rapid reporting of clinical or pre-clinical studies on a range of neuropsychiatric and neurological disorders. This journal is indexed on PubMed Central, the 'PsycINFO' database and CAS,

and is the official journal of The International Neuropsychiatric Association (INA). The manuscript management system is completely online and includes a very quick and fair peer-review system, which is all easy to use. Visit http://www.dovepress.com/testimonials.php to read real quotes from published authors.

Submit your manuscript here: http://www.dovepress.com/neuropsychiatric-disease-and-treatment-journal 